\title{
Classical and quantum filaments in the ground state of trapped dipolar Bose gases
}

\author{
Fabio Cinti* \\ National Institute for Theoretical Physics (NITheP), Stellenbosch 7600, South Africa and Institute of Theoretical Physics, Stellenbosch \\ University, Stellenbosch 7600, South Africa \\ Massimo Boninsegni \\ Department of Physics, University of Alberta, Edmonton, Alberta, Canada T6G 267
}

(Received 29 March 2017; published 26 July 2017)

\begin{abstract}
We study, by quantum Monte Carlo simulations, the ground state of a harmonically confined dipolar Bose gas with aligned dipole moments and with the inclusion of a repulsive two-body potential of varying range. Two different limits can clearly be identified, namely, a classical one in which the attractive part of the dipolar interaction dominates and the system forms an ordered array of parallel filaments and a quantum-mechanical one, wherein filaments are destabilized by zero-point motion, and eventually the ground state becomes a uniform cloud. The physical character of the system smoothly evolves from classical to quantum mechanical as the range of the repulsive two-body potential increases. An intermediate regime is observed in which ordered filaments are still present, albeit forming different structures from the ones predicted classically; quantum-mechanical exchanges of indistinguishable particles across different filaments allow phase coherence to be established, underlying a global superfluid response.
\end{abstract}

DOI: 10.1103/PhysRevA.96.013627

\section{INTRODUCTION}

Spatially confined assemblies of dipolar gases are the subject of ongoing experimental and theoretical investigations, mainly driven by the search for novel phases of matter that may arise as a result of the long-ranged anisotropic character of the interparticle interaction [1]. In particular, the experimental achievement of Bose-Einstein condensation (BEC) of atomic systems with large magnetic moments [2-10] suggests the intriguing possibility of identifying and studying experimentally phases simultaneously displaying ferromagnetism and superfluidity.

The peculiar features of the interaction between two electric or magnetic dipoles bring about issues of stability of a three-dimensional BEC. Specifically, if all dipoles are aligned and the BEC is elongated in the same direction of the dipoles, then the dipolar interactions are mostly attractive, leading to the collapse of the BEC, much like in a generic Bose system with purely attractive interactions. This effect, predicted at the mean-field level, was observed experimentally in a trapped assembly of chromium atoms [11]. A different outcome was reported recently [12], however, in a similar experiment involving dysprosium (Dy) in which the collapse was replaced by the formation of ordered arrays of droplets, reminiscent of the Rosenweig instability in classical ferrofluids [13,14]. In a subsequent experiment with erbium, a smooth evolution of the system from a trapped BEC to a dense macrodroplet was observed as the $s$-wave scattering length of the atomic interaction was varied [15].

It initially was proposed that the failure of the Dy BEC to collapse and the ensuing appearance of crystals of droplets, each comprising a significant fraction of all particles in the gas, may be underlain by interactions involving triplets of atoms $[16,17]$; experimental evidence [18] and theoretical arguments

\footnotetext{
*cinti@sun.ac.za
}

[19-22], however, point to both effects arising from the mere presence of a repulsive core at short distances in the interaction among pairs of atoms. Indeed, recent quantum Monte Carlo (QMC) studies have yielded evidence of both the stability of a trapped dipolar BEC against collapse [23] as well as the appearance of droplets [24] if a short-range repulsion is added to the pairwise dipolar interaction.

The physical origin of such a repulsive term could be different, depending on the physical system that one is considering; certainly any naturally occurring atomic or molecular interaction features a hard-core repulsion at short distances arising from the Pauli exclusion principle, which prevents electronic clouds of different atoms from overlapping spatially. The effective hard-core diameter in that case is on the order of a few angstroms, i.e., much smaller than the typical value of the characteristic dipolar length (see below) in the majority of current experiments with cold dipolar atoms or molecules. Significantly greater ranges could be achieved, e.g., by means of the Feshbach resonance [25].

An interesting question that remains open is whether this system may be a potential candidate for the experimental realization of a supersolid phase, namely, one possessing superfluid properties while simultaneously spontaneously breaking translational invariance [26]. A number of theoretical suggestions has been made of specific cold atom systems and settings wherein this elusive phase of matter may be observed unambiguously, for example, with Rydberg atoms [27-33]; experimentally, evidence of novel phases displaying density ordering and superfluidity has recently been reported for atomic BECs featuring spin-orbit interactions [34] or coupled to the modes of optical cavities [35].

We report here results of an extensive theoretical investigation, based on QMC simulations, of the ground state of a harmonically confined assembly of dipolar spin-zero bosons. We adopted the same microscopic model utilized in Ref. [24], including a hard-core repulsive term and with parameters of the harmonic confining potential chosen to be close to those of 
typical experimental setups. We explore the different physics that emerge as the number of particles in the harmonic trap and the range of the short-distance repulsion are varied.

The results of the simulations show that, although one cannot properly speak of the phase of a finite system, there is a clear evolution from a classical to a quantum regime as the effective diameter of the hard-core repulsion is increased. Specifically, in the limit of vanishing hard-core diameter the energetics of the system is dominated by the potential energy, namely, the attractive part of the dipolar interaction; the breakdown at low temperatures of the uniform gas into parallel filaments forming ordered structures can thus be understood easily in classical terms. In this regime, quantum-mechanical exchanges of indistinguishable particles across filaments are absent.

On the other hand, as the range of the hard-core repulsion approaches a significant fraction (i.e., a few tenths) of the characteristic length of the dipolar interaction, quantummechanical effects of zero-point motion, as well as exchanges of indistinguishable particles, first cause filaments to thicken, becoming more similar to prolate droplets, then to merge, forming ordered structures that are different from those predicted classically, and eventually to disappear altogether, the ground state becoming a uniform BEC (i.e., a single dilute cloud), consistent with the experimental findings of Ref. [15]. In the intermediate regime, henceforth referred to as "of quantum filaments," exchanges of particles across different filaments underlie a global superfluid response, similar to what is observed in supersolid droplet crystals [27].

It needs be mentioned that our results are in qualitative and quantitative disagreement with those reported in Ref. [24]; most significantly, whereas it is contended therein that the ground state of the system features ordered arrangements of filaments (in this regime, prolate droplets may be a more appropriate term) in a narrow range of values of the relevant interaction parameter (which, as stated above, is simply the characteristic diameter of the short-distance repulsive core), we show here that, for a sufficient number of particles, the breakdown into filaments occurs whenever this parameter is small compared to the natural length of the dipolar interaction. Indeed, as mentioned above such a breakdown inevitably occurs in the classical limit (i.e., of a vanishing hard-core diameter). Although the results shown here pertain to a specific choice of geometry of the confining trap, one similar to that adopted in most experiments, the basic physics illustrated here ought not depend on the details of the confinement.

The remainder of this article is organized as follows: In Sec. II we introduce the microscopic model, whereas in Sec. III we offer details of the calculation carried out in this paper; in Sec. IV we illustrate our results, outlining our conclusion in Sec. V.

\section{MODEL}

We consider an ensemble of $N$ Bose particles of spin zero, mass $m$, and dipole moment $D$, moving in three dimensions in the presence of a harmonic confining potential centered at the origin. All dipole moments are aligned, pointing in the positive $z$ direction. Henceforth, we express all lengths in terms of the characteristic length of the dipolar interaction, namely, $a \equiv m D^{2} / \hbar^{2}$, whereas $\epsilon \equiv\left(D^{2} / a^{3}\right)=\hbar^{2} /\left(m a^{2}\right)$ is the unit of energy and temperature (i.e., we set the Boltzmann constant as $k_{B}=1$ ).

The Hamiltonian of the system in dimensionless units reads as follows:

$$
\hat{H}=-\frac{1}{2} \sum_{i} \nabla_{i}^{2}+\frac{1}{2 \xi^{4}}\left(x_{i}^{2}+y_{i}^{2}+\gamma^{2} z_{i}^{2}\right)+\sum_{i<j} U\left(\mathbf{r}_{i}, \mathbf{r}_{j}\right)
$$

where $\mathbf{r}_{i} \equiv\left(x_{i}, y_{i}, z_{i}\right)$ is the position of the $i$ th particle, $\xi$ is the confining length of the harmonic trap, and $\gamma$ is a trap elongation factor in the $z$ direction; in this paper, we choose the same values of these parameters utilized in Ref. [24], namely, $\xi=$ 1.2 and $\gamma=4.0$, i.e., the trap is squeezed in the $z$ direction. This choice is aimed at rendering the geometry quantitatively close to that of most experiments.

The interaction potential $U$ between any two particles in the trap is given by

$$
U\left(\mathbf{r}, \mathbf{r}^{\prime}\right)=U_{\mathrm{sr}}\left(\left|\mathbf{r}-\mathbf{r}^{\prime}\right|\right)+V_{d}\left(\mathbf{r}, \mathbf{r}^{\prime}\right)
$$

where $V_{d}$ is the dipolar interaction which, since all dipole moments are aligned in the $z$ direction, is expressed readily as follows:

$$
V_{d}\left(\mathbf{r}, \mathbf{r}^{\prime}\right)=\frac{1}{\left|\mathbf{r}-\mathbf{r}^{\prime}\right|^{3}}\left(1-\frac{3\left(z-z^{\prime}\right)^{2}}{\left|\mathbf{r}-\mathbf{r}^{\prime}\right|^{2}}\right),
$$

whereas $U_{\text {sr }}$ is the short-distance repulsive potential, which in this paper is modeled as in Ref. [24], namely,

$$
U_{\mathrm{sr}}(r)=\left(\frac{\sigma}{r}\right)^{12}
$$

The expression (4) is that of a potential steeply rising for distances $\lesssim \sigma$; it cannot be given a rigorous theoretical justification and is adopted here for computational convenience. Its purpose is merely that of imparting a short-distance repulsion to the pairwise interaction, thereby preventing particles from "falling" onto one another (with the ensuing collapse of the system) as a result of the dipolar attraction that they experience as they approach one another along the $z$ direction. The parameter $\sigma$ does not, to our knowledge, have a direct relationship with the experimentally measured scattering length but can nevertheless be roughly regarded as a hard-core diameter.

The dipolar potential $V_{d}$ is anisotropic; it is purely repulsive (decaying as $1 / r^{3}$ ) for two particles lying on a plane perpendicular to the direction of dipole alignment, and it is most attractive for two particles approaching one another head-on along that same direction. Figure 1 shows the resulting potential on including the repulsive part [Eq. (4)]. As $\sigma \rightarrow 0$, the attractive well becomes increasingly deep, leading to the conclusion that the potential energy will dominate in this limit, and the behavior of the system will be classical.

The potential-energy minimum is achieved by "piling up" particles along the $z$ direction, i.e., forming a one-dimensional (1D) filament with an interparticle separation approximately given by $\sigma^{4 / 3}$; in the presence of a confining potential, however, as $N$ grows confinement along $z$ makes it energetically advantageous for the system at some point to start forming separate filaments. Two finite rigid parallel filaments can straightforwardly be shown to exert a repulsive force on 


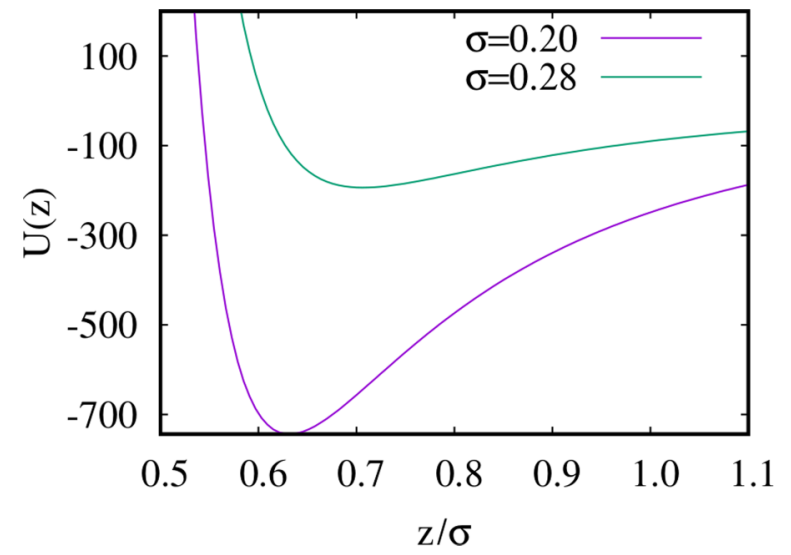

FIG. 1. Interaction potential (in the energy unit $\epsilon$ defined in the text) between two particles approaching one another head-on along the direction of alignment of the dipole moments $(z)$ for two values of the hard-core parameter $\sigma$. Potential-energy values are in units of $\epsilon$.

one another, and therefore the classical ground state of the system for large $N$ consists of filaments arranged in ordered structures, similar to those formed by, e.g., dipolar particles in a two-dimensional harmonic trap [36]. A few examples of such structures are shown in the top part of Fig. 2.

For a given trap geometry as well as number $N$ of particles and value of $\sigma$, it is a straightforward exercise to identify the classical ground states by direct minimization of the potential energy. Figure 2 shows the number $N_{f}$ of filaments in the classical ground state of (1) as a function of $N$ for the trap geometry adopted here. As we can see, $N_{f}$ is an increasing function of $N$, more rapidly so the greater $\sigma$, as fewer and

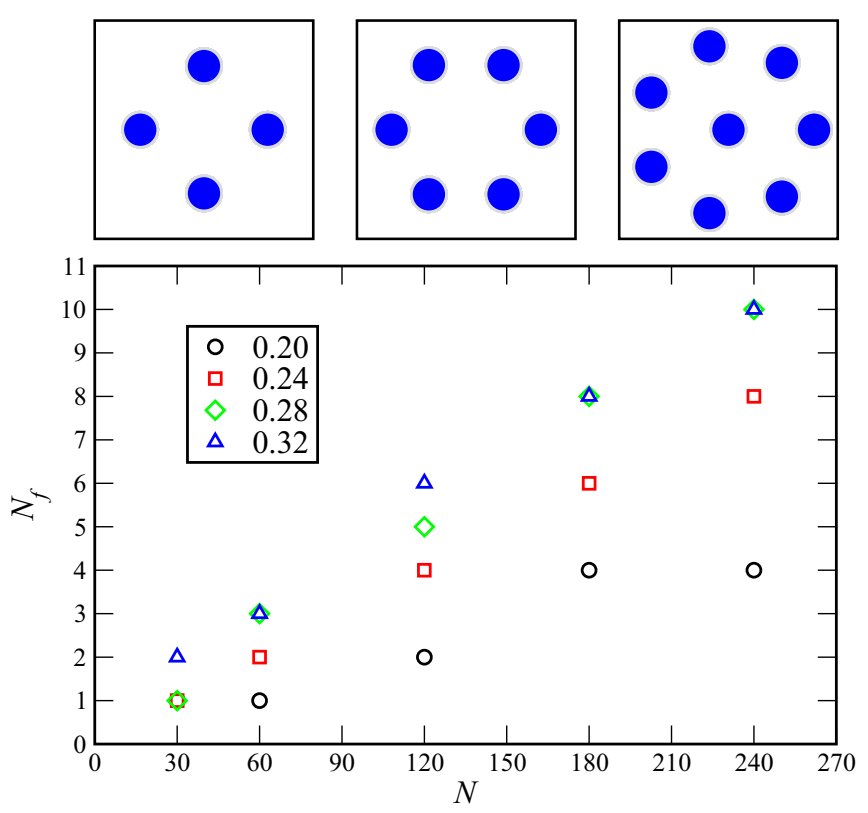

FIG. 2. Top: Typical crystalline structures formed by filaments (top view). Bottom: Number of filaments in the classical ground state of model (1) as a function of total particle number $N$ for different values of $\sigma$. fewer particles can be accommodated in a single filament due to the increased 1D lattice constant.

As mentioned above, the classical picture should hold in the limit $\sigma \rightarrow 0$ as the attractive well of the anisotropic pairwise potential grows deeper. On the other hand, as $\sigma$ increases and becomes of order one (i.e., comparable to the dipolar length $a$ ), the well is shallow, and one can expect quantum zero-point motion as well as exchanges of indistinguishable particles [37] to destabilize the classical filaments in favor of a uniform cloud or perhaps different ordered structures. In order to investigate this scenario and in particular to account in full for quantum-mechanical effects, we adopted first-principles numerical (QMC) simulations.

\section{METHODOLOGY}

The low-temperature phase diagram of the system described by Eq. (1) as a function of the total number $N$ of particles in the trap as well as of the hard-core parameter $\sigma$ has been studied in this paper by means of first-principles numerical simulations, based on the continuous-space worm algorithm [38,39]. Since this technique is by now fairly well established and extensively described in the literature, we will not review it here. We used a variant of the algorithm in which the number of particles $N$ is fixed [40,41]. Details of the simulation are mostly standard, one difference with respect to previous works is that we used here the so-called "primitive" approximation for the short imaginary time $(\tau)$ propagator, which requires a greater number of time slices than more elaborate propagators (e.g., the fourth-order one [42]) but which we found more convenient due to its simplicity when dealing with an anisotropic interaction. Obviously, all of the results presented here are extrapolated to the $\tau \rightarrow 0$ limit; in general, we found numerical estimates for structural and superfluid properties of interest here, obtained with a value of the time-step $\tau \sim 10^{-3} \epsilon^{-1}$ to be indistinguishable from the extrapolated ones within the statistical uncertainties of the calculation.

Because we are interested mainly in the physics of the system in the $T \rightarrow 0$ limit, we generally report here results corresponding to temperatures $T$ sufficiently low to regard them as essentially ground-state results, mainly because further lowering the temperature does not result in significant qualitative or quantitative changes. This typically is achieved at higher temperatures in the classical (i.e., $\sigma \rightarrow 0$ ) limit, but in general we observed that simulations carried out at a temperature $T \sim \epsilon$ can be regarded as representative of the ground state of the system.

We carried out simulations of systems typically comprising a few hundred particles, 1024 being the largest size considered here; for the trap geometry utilized, this corresponds to a density range between few tens and few hundreds of $a^{-3}$. We typically started our simulations from many-particle configurations corresponding to the classical ground states for the chosen values of $N$ and $\sigma$. This is the same protocol normally adopted when simulating, e.g., quantum crystals, which consists of placing particles initially at lattice sites consistent with the crystalline structure of interest. We also have used as a starting configuration a single filament at the center of the trap as well as a high-temperature gaslike 

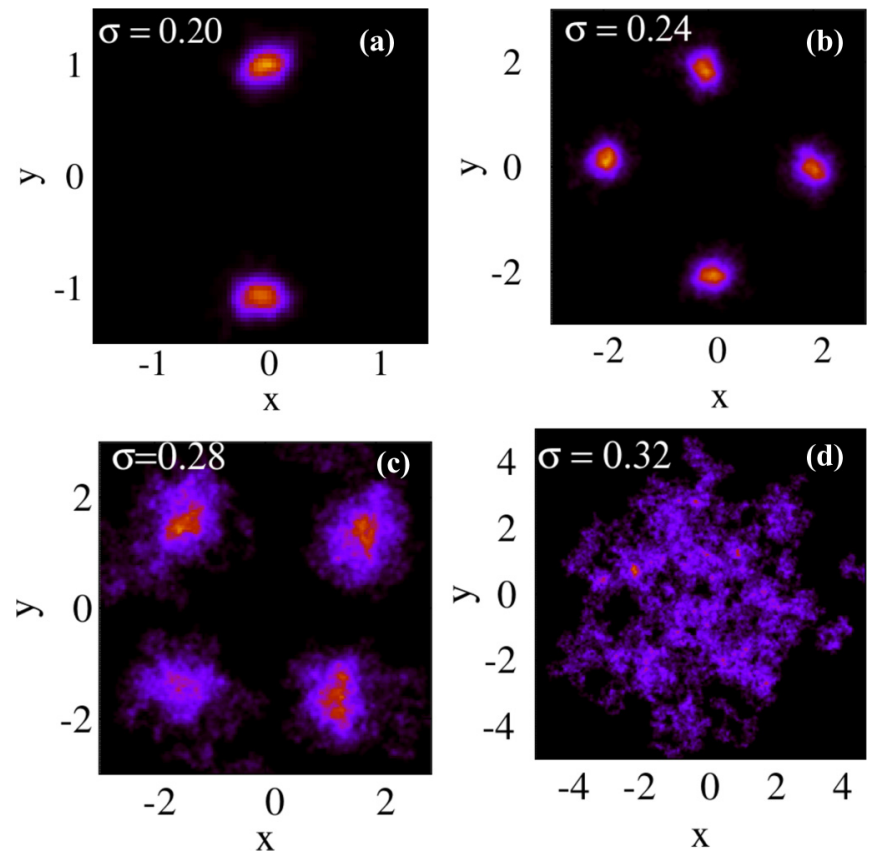

FIG. 3. Density maps for a system of $N=120$ particles (top view) for four different values of the parameter $\sigma$. All lengths are all in units of $a$ (see the text).

configuration. As we are interested primarily in the filament structure of the system, physical conclusions mainly are drawn based on a visual inspection of the many-particle configurations generated in the course of sufficiently long simulation runs.

Regarding the superfluid response, in principle it can be evaluated using the well-known "area" estimator [43], appropriate for a finite system, such as the one considered here. In practice, the filament structure of the system renders the value of the superfluid fraction comparatively low in the supersolid phase (not unexpectedly [30]), and therefore we rely on a more indirect criterion, namely, we monitor the frequency of cycles of permutations of identical particles involving a number thereof significantly greater than that in a single filament. Although there is no quantitative connection between the permutation cycles and the superfluid fraction [44] and although it is possible for individual quasi-1D filaments to possess an individual superfluid response (e.g., in the Luttinger sense), a global superfluid phase requires exchanges of particles across filaments (see, for instance, Ref. [27]).

\section{RESULTS}

We begin with a qualitative illustration of the physical trend occurring as the value of the parameter $\sigma$ is increased. Figures 3 and 4 show two-dimensional density maps (integrated over the $z$ direction) for a system comprising $N=120$ and $N=180$ particles for different values of the hard-core parameter $\sigma$, namely, $0.20,0.24,0.28$, and 0.32 . These maps are obtained from instantaneous many-particle configurations generated during a sufficiently long simulation. They are physically equivalent to all other configurations generated in the course of the same run for the specific conditions considered (e.g.,
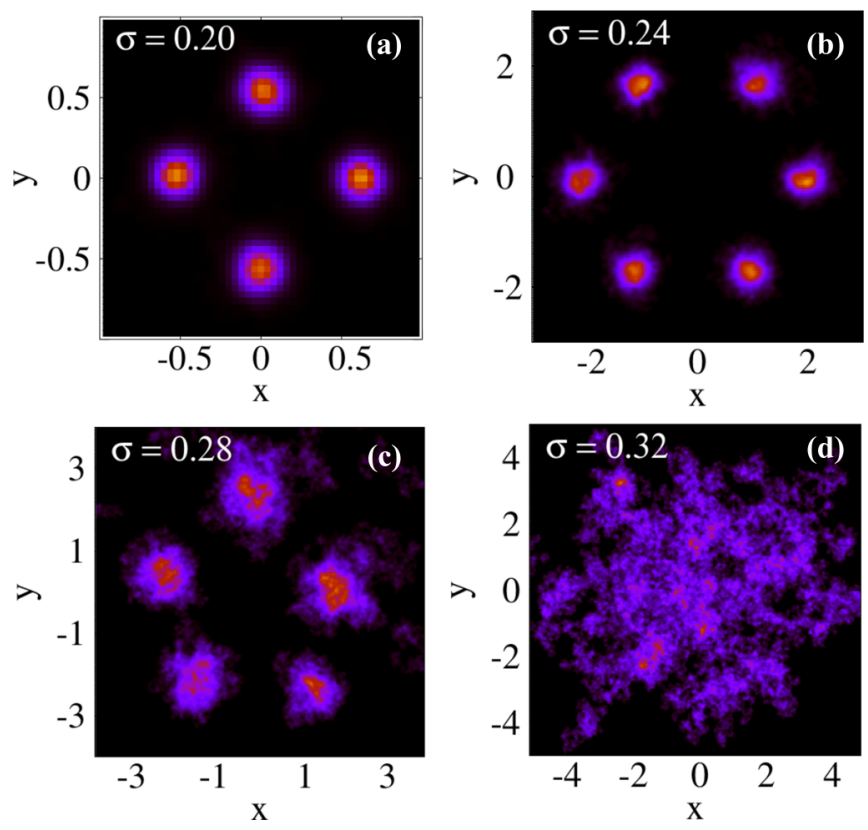

FIG. 4. The same as in Fig. 3 but for $N=180$ particles.

others differ at the most by an overall rotation) and therefore are presented as statistically representative. The temperature of the simulations is in all cases low enough that the system may be regarded in the ground state; specifically, it is $T=5 \epsilon$ for $\sigma=0.20$, whereas it is $T=\epsilon$ for all other cases. We have, however, carried out simulations down to $T=0.5 \epsilon$ and ascertained that the physics remains the same in all cases.

A filament structure is identified clearly in three out of the four cases, whereas for the largest value of $\sigma$ the system forms a uniform cloud. As we will see, however, there are substantial differences between the physical behavior at $\sigma \lesssim 0.24$ and that observed in the range of $0.24 \lesssim \sigma \lesssim 0.3$.

For values of $\sigma \lesssim 0.24$, we find that the simulated system remains very close to its starting configuration, which, as mentioned above, corresponds to the classical ground state. Quantum-mechanical zero-point motion causes the filaments to expand slightly in the transverse direction, acquiring a relatively small diameter (e.g., approximately 0.02 for $\sigma=$ 0.1 ) as illustrated in Fig. 3 for the two lower values of $\sigma$. Exchanges among particles in the same filament become relatively important at low temperatures as $\sigma$ approaches a value close to $\sim 0.2$ but do not significantly affect the structure of the filament itself. On the other hand, particle exchanges across filaments are not observed in this regime to which we refer as classical.

In such a regime, filaments always form; we also observe that, if the simulation is started from a different ordered filament arrangement, e.g., including a greater number of filaments than the classical ground state, such a configuration remains stable, albeit its energy is greater than that obtained if the classical ground state is taken as a starting point. This can be interpreted as evidence of long-lived ordered filament configurations above the ground state.

As $\sigma \rightarrow 0$, the density of particles in a single filament grows, i.e., more and more particles are needed to form more than one filament. For example, for a value of $\sigma=0.1$, the 
lowest considered in this paper, the ground state of the system consists of a single narrow filament along the axis of the trap for $N$ at least up to 1024 . This observation is consistent with the results of the QMC simulation carried out by Saito [23], based on a different model for the short-range repulsion but with a choice of the equivalent hard-core parameter well into the classical regime (a quantitative comparison of the results obtained here with those of Saito would require that $\sigma$ be set to a value less than 0.1 ). On the other hand, it was reported in Ref. [24], based on a ground-state QMC calculation making use of the same microscopic Hamiltonian adopted here, that filaments (prolate droplets) only form in the narrow $0.24<$ $\sigma<0.28$ range, which is clearly in disagreement with the arguments furnished and the results obtained here. We come back to this point below.

As the hard-core parameter $\sigma$ grows above a value of $\sim 0.24$, the physics of the system changes qualitatively. Quantum zeropoint motion and permutations of indistinguishable particles cause filaments to melt and/or merge, and for $\sigma \gtrsim 0.3$ the ground state is a uniform cloud (100\% superfluid in the $T \rightarrow 0$ limit, the superfluid response uniform across the cloud as we have ascertained through the computation of the superfluid density using the area estimator [44]) with no remnant of filaments as shown in panels (d) of Figs. 3 and 4. There exists, however, an interesting intermediate regime around $\sigma=0.28$ wherein the system displays an ordered filament structure that is different from the classical one.

Consider for definiteness the case of $N=120, \sigma=0.28$, shown in panel (c) of Fig. 3. Four lumps can clearly be identified; although the simulation initially was started from the classical ground state, including five filaments (as shown in Fig. 2), we observe these filaments to merge in a single cloud, which then divides into the four prolate droplets shown in Fig. 3. Remarkably, this is observed consistently in this system, at sufficiently low $T(\lesssim \epsilon)$, regardless of the initial configuration chosen. Indeed, four droplets form even if the simulation is started from a single filament at the center of the trap, or, e.g., three filaments, or from a disordered high-temperature initial configuration; for example, the result shown in Fig. 3 for $\sigma=0.28$ pertains to a simulation in which particles initially were placed on a single filament. We therefore conclude that the presence of four droplets is an intrinsic quantum-mechanical signature of the ground state of the system for this number of particles and this value of $\sigma$. These quantum droplets are not pinned at classical locations but rather are observed to meander in the course of the simulation; their relative positions, however, remain fairly constant. The same behavior is observed in the simulation of the system comprising $N=180$ particles for this value of $\sigma$, which forms five droplets at low $T$ irrespective of the starting configuration. It should be noted that, in this regime to which we refer as quantum, exchanges of identical particles are not restricted to individual droplets but extend across different droplets.

The number of droplets in the ground state is consistently less than that predicted classically as a result of consolidation arising from quantum exchanges, which results in an effective attraction among particles obeying Bose statistics. For example, in the case of $\sigma=0.28$ and $N=180$ the number of droplets in the ground state is five as opposed to the eight

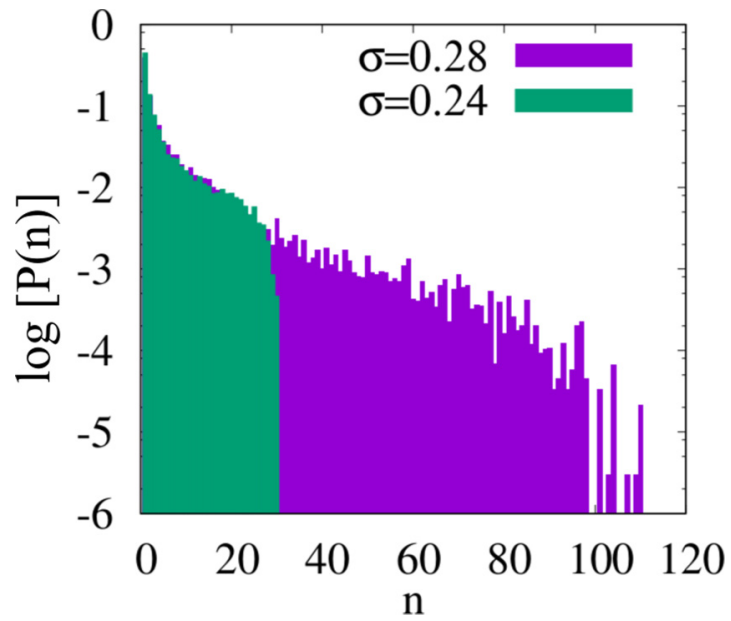

FIG. 5. Logarithm (base 10) of frequency of occurrence of cycles comprising $n$ particles at low $T$ (see the text) for a system of $N=120$ particles and for two different values of the parameter $\sigma$.

predicted classically (see Fig. 2). Altogether, the formation of droplets in this regime is strikingly reminiscent of the exchange-induced crystallization already observed in a system of soft-core bosons [45].

As mentioned in the Introduction, one of the interesting questions about this particular system is whether its lowtemperature phase diagram may include a supersolid phase; specifically, one may imagine a superfluid phase consisting of ordered filaments, each one individually superfluid, wherein phase coherence is established through particle exchanges across filaments. The results obtained in this paper show that such a phase does not occur in what we refer to as the classical regime (i.e., $\sigma \lesssim 0.24$ ) wherein filaments are spaced apart and particle exchanges only take place within filaments. Even though individual filaments can be quasi-1D superfluids, the phase as a whole cannot acquire global phase coherence. On the other hand, in the quantum filament regime (roughly $0.24<\sigma<0.3$ ), particle exchanges across filaments are observed, leading us to make the conclusion that a supersolid phase indeed exists in this regime.

To illustrate this point, Fig. 5 shows the (logarithm of the) computed frequency $P(n)$ of the occurrence of permutation cycles involving $n$ particles $(1 \leqslant n \leqslant N)$ for a system of $N=120$ particles. The distributions shown pertain to two different values of $\sigma$, namely, 0.24 (in this case, the temperature is $T=\epsilon)$ and $0.28(T=0.5 \epsilon)$. The important point here is that the ground state features the same number of filaments (four) in both cases and the qualitative features of the two distributions do not change at lower temperatures. Now, whereas the $P(n)$ for $\sigma=0.24$ abruptly falls to zero for $n>30$, which is the number of particles in a single filament, that for $\sigma=0.28$ features cycles involving almost all particles in the system. As mentioned above, the presence of cycles involving all particles in a single filament for $\sigma=0.24$ makes it likely that filaments are superfluid (again in the Luttinger sense as these are quasi-1D objects); however, it is for $\sigma \sim 0.28$ that a supersolid phase is predicted as the system still features an ordered array of filaments while at the same time displaying exchanges across filaments. Consistently, the superfluid response, evalu- 
ated with the area estimator, yields a finite value of approximately $20 \%$ at $T=0.5$ [46]. This is the same physics observed in the supersolid cluster crystal phase [27,30]. On the other hand, for $\sigma \leqslant 0.24$ the character of the system at low $T$ is that of an array of independent quasi-1D superfluids.

\section{DISCUSSION}

In this paper we carried out an extensive theoretical investigation of the low-temperature (ground-state) properties of a harmonically confined assembly of dipolar bosons with dipole moments all aligned. Our paper is based on firstprinciples numerical simulations, and we adopted the same theoretical description recently utilized in another study, which includes, besides the dipolar interaction, a short-range repulsive potential to prevent the system from collapsing. Although we have made use of a specific trap geometry, chosen to mimic as closely as possible that of most experiments, the results presented here are general enough that they should be independent of that aspect. As well, the specific way in which we have modeled the short-range repulsion ought not affect the most important qualitative and quantitative conclusions drawn here. Indeed, the main physical effect that we set out to explore, namely, the formation of a ordered arrays of filaments, appears observable in a rather wide range of physical conditions, considerably more extended than suggested in Ref. [24].

In particular, in the limit in which the range of the hard-core short-distance repulsion is small compared to the characteristic length of the dipolar interaction, the system forms filaments as a result of a purely classical effect, arising from the competition between the attractive part of the dipolar potential and the confinement. As this limit is the one relevant to most current experimental settings, the observation of droplets in experiments is not altogether surprising. The physical character of the filaments evolve as the hard-core diameter increases, causing a weakening of the dipolar attraction. Whereas for a sufficiently large value of the hard-core diameter filaments melt, and the ground state of the system is a uniform cloud of particles, we have found that there exists an intermediate region that has no classical counterpart wherein filaments form due to purely quantum effects, namely, exchanges of indistinguishable particles obeying Bose statistics. These filaments form differently ordered structures from those predicted classically, which can give rise to a supersolid phase as demonstrated by the simulation results shown here, underlain by particle exchanges across filaments. Whether these physical conditions, chiefly a hard-core repulsion of range approximately one-third of the characteristic dipolar length, are experimentally achievable at this time, remains to be ascertained.

Our results are at variance with those of Macia et al. (Ref. [24]) not only regarding the number of droplets that form in the ground state of the system for specific values of $\sigma$ and $N$, but also, much more significantly, because they ostensibly failed to observe any filament phase in the classical region where they most obviously should occur, based on the simple (potential-energy) considerations outlined above. In their study, they employed the $T=0$ path-integral ground-state method $[47,48]$, which is in principle capable of projecting the true ground state of a Bose system out of an initial nonorthogonal trial wave function. It need be noted, however, that the trial wave function utilized in that study is just a constant, i.e., it has very small overlap with a ground state consisting of a few quasi-1D filaments; it is therefore conceivable that the projection algorithm may need an exceedingly long time to converge to the true ground state, especially in a regime dominated by the potential energy. This is akin to the convergence problem that one would face on attempting to extract a crystalline ground state from a noninteracting variational ansatz (i.e., a constant trial wave function), something in principle possible but usually unfeasible in practice. More generally, although zero-temperature methods prove useful in specific cases, the experience accumulated over the past two decades points to finite-temperature techniques as being typically more reliable, at least for Bose systems [49].

\section{ACKNOWLEDGMENTS}

This work was supported, in part, by the Natural Sciences and Engineering Research Council of Canada (NSERC). M.B. gratefully acknowledges the hospitality of the National Institute for Theoretical Physics (NITheP), Stellenbosch where most of this research work was carried out. The authors wish to acknowledge useful conversations with A. Kuklov, T. Macrí, and S. Moroni. Computing support from Westgrid also is acknowledged.
[1] See, e.g., T. Lahaye, C. Menotti, L. Santos, M. Lewenstein, and T. Pfau, Rep. Prog. Phys. 72, 126401 (2009), and references therein.

[2] A. Griesmaier, J. Werner, S. Hensler, J. Stuhler, and T. Pfau, Phys. Rev. Lett. 94, 160401 (2005).

[3] M. Lu, N. Q. Burdick, S. H. Youn, and B. L. Lev, Phys. Rev. Lett. 107, 190401 (2011).

[4] K. Aikawa, A. Frisch, M. Mark, S. Baier, A. Rietzler, R. Grimm, and F. Ferlaino, Phys. Rev. Lett. 108, 210401 (2012).

[5] A. de Paz, A. Sharma, A. Chotia, E. Maréchal, J. H. Huckans, P. Pedri, L. Santos, O. Gorceix, L. Vernac, and B. Laburthe-Tolra, Phys. Rev. Lett. 111, 185305 (2013).
[6] K.-K. Ni, S. Ospelkaus, M. H. G. de Miranda, A. Peér, B. Neyenhuis, J. J. Zirbel, S. Kotochigova, P. S. Julienne, D. S. Jin, and J. Ye, Science 322, 231 (2008).

[7] B. Yan, S. A. Moses, B. Gadway, J. P. Covey, K. R. A. Hazzard, A. M. Rey, D. S. Jin, and J. Ye, Nature (London) 501, 521 (2013).

[8] T. Takekoshi, L. Reichsöllner, A. Schindewolf, J. M. Hutson, C. R. Le Sueur, O. Dulieu, F. Ferlaino, R. Grimm, and H.-C. Nägerl, Phys. Rev. Lett. 113, 205301 (2014).

[9] J. W. Park, S. A. Will, and M. W. Zwierlein, Phys. Rev. Lett. 114, 205302 (2015).

[10] J. B. Balewski, A. T. Krupp, A. Gaj, S. Hofferberth, R. Löw, and T. Pfau, New J. Phys. 16, 063012 (2014). 
[11] T. Lahaye, J. Metz, B. Fröhlich, T. Koch, M. Meister, A. Griesmaier, T. Pfau, H. Saito, Y. Kawaguchi, and M. Ueda, Phys. Rev. Lett. 101, 080401 (2008).

[12] H. Kadau, M. Schmitt, M. Wenzel, C. Wink, T. Maier, I. FerrierBarbut, and T. Pfau, Nature (London) 530, 194 (2016).

[13] M. D. Cowley and R. E. Rosensweig, J. Fluid Mech. 30, 671 (1967).

[14] V. I. Timonen, M. Latikka, L. Leibler, R. H. A. Ras, and O. Ikkala, Science 341, 253 (2013).

[15] L. Chomaz, S. Baier, D. Petter, M. J. Mark, F. Wächtler, L. Santos, and F. Ferlaino, Phys. Rev. X 6, 041039 (2016).

[16] K.-T. Xi and H. Saito, Phys. Rev. A 93, 011604 (2016).

[17] R. N. Bisset and P. B. Blakie, Phys. Rev. A 92, 061603 (2015).

[18] I. Ferrier-Barbut, H. Kadau, M. Schmitt, M. Wenzel, and T. Pfau, Phys. Rev. Lett. 116, 215301 (2016).

[19] D. S. Petrov, Phys. Rev. Lett. 115, 155302 (2015).

[20] F. Wächtler and L. Santos, Phys. Rev. A 93, 061603 (2016).

[21] F. Wächtler and L. Santos, Phys. Rev. A 94, 043618 (2016).

[22] R. N. Bisset, R. M. Wilson, D. Baillie, and P. B. Blakie, Phys. Rev. A 94, 033619 (2016).

[23] H. Saito, J. Phys. Soc. Jpn. 85, 053001 (2016).

[24] A. Macia, J. Sánchez-Baena, J. Boronat, and F. Mazzanti, Phys. Rev. Lett. 117, 205301 (2016).

[25] See, for instance, C. J. Pethick and H. Smith, Bose-Einstein Condensation in Dilute Gases (Cambridge University Press, Cambridge, UK, 2005).

[26] M. Boninsegni and N. Prokof'ev, Rev. Mod. Phys. 84, 759 (2012).

[27] F. Cinti, P. Jain, M. Boninsegni, A. Micheli, P. Zoller, and G. Pupillo, Phys. Rev. Lett. 105, 135301 (2010).

[28] S. Saccani, S. Moroni, and M. Boninsegni, Phys. Rev. B 83, 092506 (2011).

[29] S. Saccani, S. Moroni, and M. Boninsegni, Phys. Rev. Lett. 108, 175301 (2012).

[30] M. Boninsegni, J. Low Temp. Phys. 168, 137 (2011).
[31] T. Macrì, F. Maucher, F. Cinti, and T. Pohl, Phys. Rev. A 87, 061602(R) (2013).

[32] T. Macrì, S. Saccani, and F. Cinti, J. Low Temp. Phys. 175, 631 (2014)

[33] F. Cinti, T. Macrì, W. Lechner, G. Pupillo, and T. Pohl, Nat. Commun. 5, 3235 (2014).

[34] J.-R. Li et al., Nature (London) 543, 91 (2017).

[35] J. Léonard, A. Morales, P. Zupancic, T. Esslinger, and T. Donner, Nature (London) 543, 87 (2017).

[36] M. Boninsegni, Phys. Rev. A 87, 063604 (2013).

[37] M. Boninsegni, L. Pollet, N. Prokof'ev, and B. Svistunov, Phys. Rev. Lett. 109, 025302 (2012).

[38] M. Boninsegni, N. Prokof'ev, and B. Svistunov, Phys. Rev. Lett. 96, 070601 (2006).

[39] M. Boninsegni, N. V. Prokof'ev, and B. V. Svistunov, Phys. Rev. E 74, 036701 (2006).

[40] F. Mezzacapo and M. Boninsegni, Phys. Rev. Lett. 97, 045301 (2006).

[41] F. Mezzacapo and M. Boninsegni, Phys. Rev. A 75, 033201 (2007).

[42] See, for instance, M. Boninsegni, J. Low Temp. Phys. 141, 27 (2005).

[43] P. Sindzingre, M. L. Klein, and D. M. Ceperley, Phys. Rev. Lett. 63, 1601 (1989).

[44] See, for instance, F. Mezzacapo and M. Boninsegni, Phys. Rev. Lett. 100, 145301 (2008).

[45] F. Cinti, M. Boninsegni, and T. Pohl, New J. Phys. 16, 033038 (2014).

[46] The superfluid fraction ought not saturate to $100 \%$ even at $T=0$ in a phase that simultaneously breaks rotational invariance.

[47] A. Sarsa, K. E. Schmidt, and W. R. Magro, J. Chem. Phys. 113, 1366 (2000).

[48] J. E. Cuervo, P.-N. Roy, and M. Boninsegni, J. Chem. Phys. 122, 114504 (2005).

[49] See, for instance, M. Boninsegni, Phys. Rev. Lett. 111, 235303 (2013). 